\title{
Damage accumulation in silica glass nanofibers
}

\author{
Silvia Bonfanti, ${ }^{\dagger, \delta}$ Ezequiel E. Ferrero, ${ }^{,+\dagger, \downarrow}$ Alessandro L. Sellerio, ${ }^{\dagger}$ \\ Roberto Guerra, ${ }^{\dagger}$ and Stefano Zapperi, ${ }^{*,+}$,
}

$\dagger$ Center for Complexity and Biosystems, Department of Physics, University of Milano, via Celoria 16, 20133 Milano, Italy

$\ddagger$ CONICET, Centro Atómico Bariloche, Av. Bustillo 9500, 8400 S. C. de Bariloche, Río

Negro, Argentina

๑ Consiglio Nazionale delle Ricerche, Istituto di Chimica della Materia Condensata e di Tecnologie per l'Energia, Via R. Cozzi 53, 20125 Milano, Italy

$\S$ Contributed equally to this work

E-mail: ferrero@cab.cnea.gov.ar; stefano.zapperi@unimi.it

\begin{abstract}
The origin of the brittle-to-ductile transition, experimentally observed in amorphous silica nanofibers as the sample size is reduced, is still debated. Here we investigate the issue by extensive molecular dynamics simulations at low and room temperatures for a broad range of sample sizes, with open and periodic boundary conditions. Our results show that small samplesize enhanced ductility is primarily due to diffuse damage accumulation, that for larger samples leads to brittle catastrophic failure. Surface effects such as boundary fluidization contribute to ductility at room temperature by promoting necking, but are not the main driver of the transition. Our results suggest that the experimentally observed size-induced ductility of
\end{abstract}




\section{silica nanofibers is a manifestation of finite-size criticality, as expected in general for quasi-brittle disordered networks.}

\section{Keywords}

silica, nanowires, nanofibers, fracture, ductility

\section{Introduction}

Despite its relevance for several applications, from basic tools to nano-technology, the understanding of the mechanical response of amorphous materials is still incomplete. The long-standing engineering issue is to ensure simultaneously high strength and extended deformability. These two properties are unfortunately nearly mutually exclusive: Very strong structural materials (e.g. ceramics) can sustain enormous stresses but fail catastrophically, with no signs of plasticity, while metallic alloys are able to sustain a degree of plastic strain by sacrificing strength. We broadly distinguish two ideally-opposite kinds of mechanical failure: brittle failure where a catastrophic fracture event happens soon after the elastic deformation limit, and ductile failure involving a considerably amount of plastic deformation before failure. Between these two extremes lies the quasi-brittle behavior, observed in many disordered materials such as concrete and rocks, where failure is preceded by extensive damage accumulation. ${ }^{1}$

Traditionally, a transition from brittle to ductile failure in the same material can be achieved by decreasing the strain rate $^{2,3}$ or increasing the temperature, ${ }^{2,4,5}$ but it is also possible to reach the same goal by modifying sample preparation and composition. For ex-

ample, silica glasses quenched under increasingly high pressures show enhanced ductility, ${ }^{6}$ suggesting a role for increased defect concentration. Recent results are changing the conventional view of the brittle-to-ductile transition (BDT) suggesting that it is possible to induce ductility just by reducing the sample size towards the nanoscale, ${ }^{7-12}$ as demonstrated for 
silica glass nanofibers under tensile load. ${ }^{9,10}$

The physical origin of this sample-size induced BDT in silica glasses is currently debated. The main physical mechanism invoked to explain the onset of ductility at the nanoscale involves the interplay between surface and bulk effects, whose relative importance clearly changes with the sample size. This appears to be a widely observed phenomenon, manifested not only in amorphous solids, ${ }^{13-16}$ but, to some extent, also in crystals. ${ }^{14,17,18} \mathrm{~A}$ "fluid-like" surface layer similar to those observed in glassy polymers ${ }^{19,20}$ was reported in recent experiments on amorphous silica. ${ }^{9,10}$ The idea is that when stress is applied, surface atoms (and group of atoms) more mobile than in the bulk due to lower bond coordination, would be the first to switch bonds and rearrange or even migrate along the surface. As the fiber diameter decreases, this surface effect is expected to have a growing influence in the deformation process with respect to bulk processes like crack nucleation and propagation.

To shed light on the mechanism leading to the observed BDT in silica glass, we perform extensive molecular dynamics simulations. The advantages of this approach are the direct visualization of microscopic bond breaking and the possibility to compare open and periodic boundary conditions to highlight the role of the free surface. Our results demonstrate that bond breaking plays a central role for the enhanced small-size ductility, while free surfaces only play a role at high temperatures.

\section{Results}

\section{Low temperature deformation of amorphous silica glass nanowires}

We simulate the tensile deformation of cylindric nanofibers of amorphous silica glass $(a$ $\mathrm{SiO}_{2}$ ) using different sample sizes with open boundary condition (obc). To disentangle the role of structural disorder from thermal fluctuations, we first perform simulations at very low temperature, $\sim 1 \mathrm{~K}$ (see Methods section for details).

Figure 1a shows typical stress-strain curves obtained from the deformation of two cylin- 


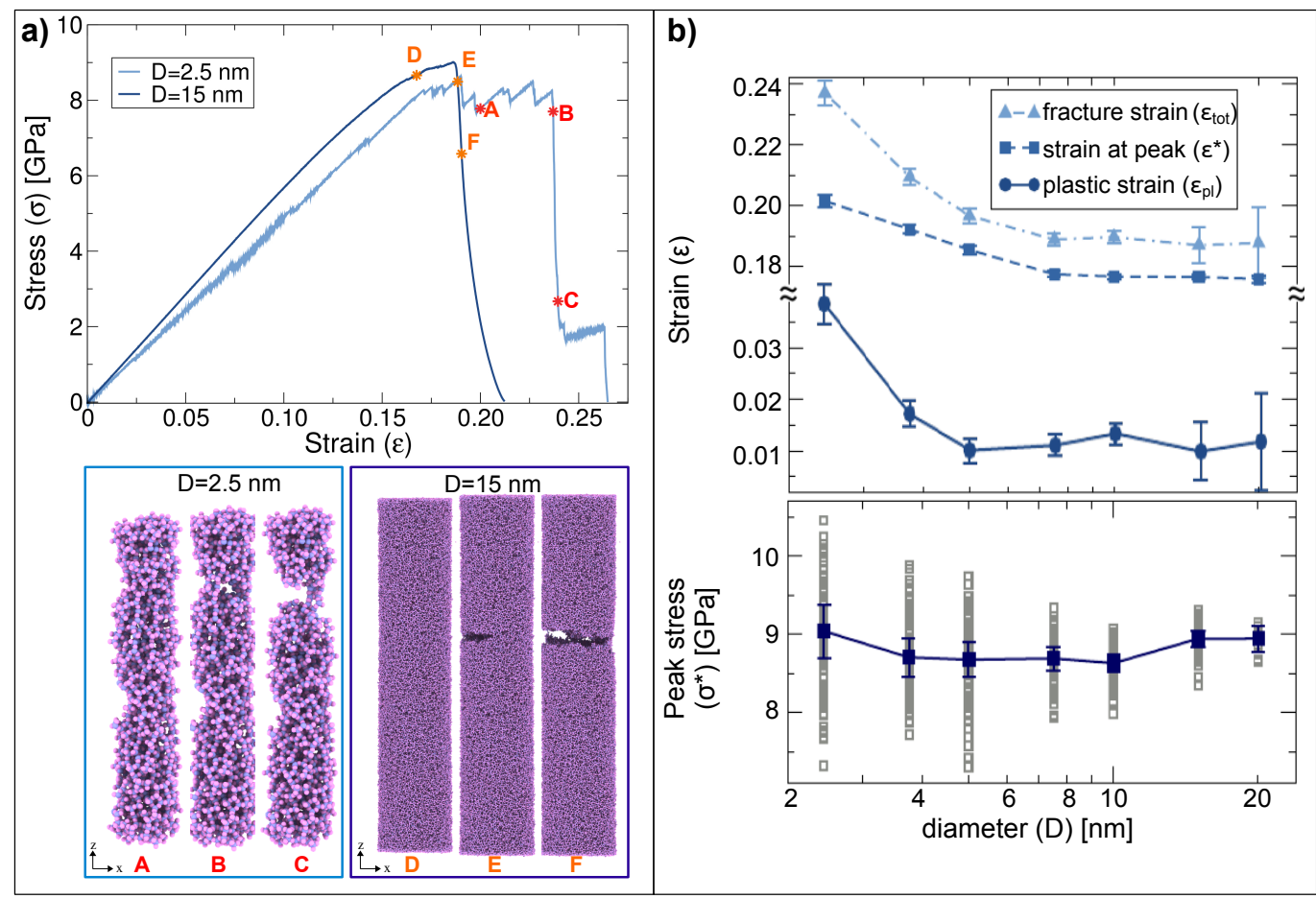

Figure 1: Brittle-to-ductile crossover as the sample size is reduced - (a) (top) Stress-strain curves of two typical samples of diameter $D=2.5 \mathrm{~nm}$ and $D=15 \mathrm{~nm}$, and (bottom) snapshots of the same nanofibers during the deformation at configurations marked by labels on the corresponding stress curves. (Violet spheres: Si atoms, pink spheres: O atoms. Arbitrary scale). See SI Movie 3 and 4 for full animations. (b) (top) Deformation up to peak stress $\epsilon^{*} \equiv \epsilon\left(\sigma_{\max }\right)$, total deformation $\epsilon_{\mathrm{tot}}$ before fracture, plastic deformation $\epsilon_{p l} \equiv\left(\epsilon_{\mathrm{tot}}-\epsilon^{*}\right)$, and (bottom) the average maximum stress $\sigma^{*}$ as a function of the sample diameter. In the latter, scattered symbols show the $\sigma^{*}$ value of each individual sample, while blue squares correspond to the average.

dric nanofibers of different size, together with visual snapshots at different applied strain during the deformation at $1 \mathrm{~K}$. For large fiber diameters, we observe an abrupt fracture right after the deviation from a purely elastic regime, signaling a brittle response also reflected by the relatively sharp crack surfaces formed in the fracture region. At very small diameters, beyond the elastic limit we observe instead a sustained stress-strain curve of jagged shape, and a more irregular crack surface with appreciable necking at the onset of fracture. We note that, differently than in metallic glass nanofibers ${ }^{11}$ where the crack is oriented at $45^{\circ}$ with respect to the loading direction, in $\mathrm{SiO}_{2}$ glasses ${ }^{9}$ the crack is oriented at $90^{\circ}$. This is due to the fact that metallic glasses fail along the direction of maximum shear, while silica glasses fail along the direction of maximum tensile stress. 
To quantify the brittle to ductile crossover, we average over several stress-strain curves four characteristic quantities: (i) the strain at which the engineer stress peaks, $\epsilon^{*}$. This would be ideally a measure of elastic elongation previous to the yielding point, but since a non-negligible - and sometimes important - plastic deformation occurs before the stress peak, this does not necessarily coincides with the plastic onset; (ii) the total strain before fracture, $\epsilon_{\mathrm{tot}}$; (iii) the plastic strain, $\epsilon_{p l} \equiv\left(\epsilon_{\mathrm{tot}}-\epsilon^{*}\right)$, (iv) the maximum stress or peak stress, $\sigma^{*}$. Fig. 1b (top panel) shows that $\epsilon^{*}$, converging to $\sim 17.5 \%$ at large diameters, starts to increase below a threshold diameter of $\sim 8 \mathrm{~nm}$ and exceeds $\sim 20 \%$ at the smallest considered size. Such increased endurable strain in the pre-yield deformation stage is in well agreement with experimental data. ${ }^{10}$ Similarly, the sample averaged $\epsilon_{\text {tot }}$ increases below the same threshold diameter from $\sim 19 \%$ up to $\sim 24 \%$, more steeply than $\epsilon^{*}$, which reflects on the plastic strain $\epsilon_{p l}$ trend.

Turning our attention to the fracture strength, the peak stress value $\sigma^{*}$, shown in Fig. 1b (bottom panel) presents a distribution of values broader for smaller samples, without an appreciable variation of the average with the system size, at least for the range of sizes we study. The experimental evidence of Luo et al. ${ }^{9}$, showing a weak strength increase at decreasing $D$ could be related to the broadening of the sample-to-sample fluctuations that we observe. Furthermore, our data clearly support a nanoscale size effect which reduces the elastic modulus $E$ at small size, suggesting a weaker contribution from the surface region (see S.I. Fig. S1).

\section{Origin of small-size ductility}

We inspect the $x-y$ mean-squared displacement of the atoms composing the smallest nanofiber at different radial positions (see S.I. Figs. S2, S3). Notice that in our simulations, regardless the sample diameter, the outer layer - defined as the layer in which the density drops from its bulk value to zero - has an approximate fixed width of $0.5 \mathrm{~nm}$ (see S.I. Fig. S4). Therefore, as we decrease the sample diameter the surface layer becomes more and more relevant. We 


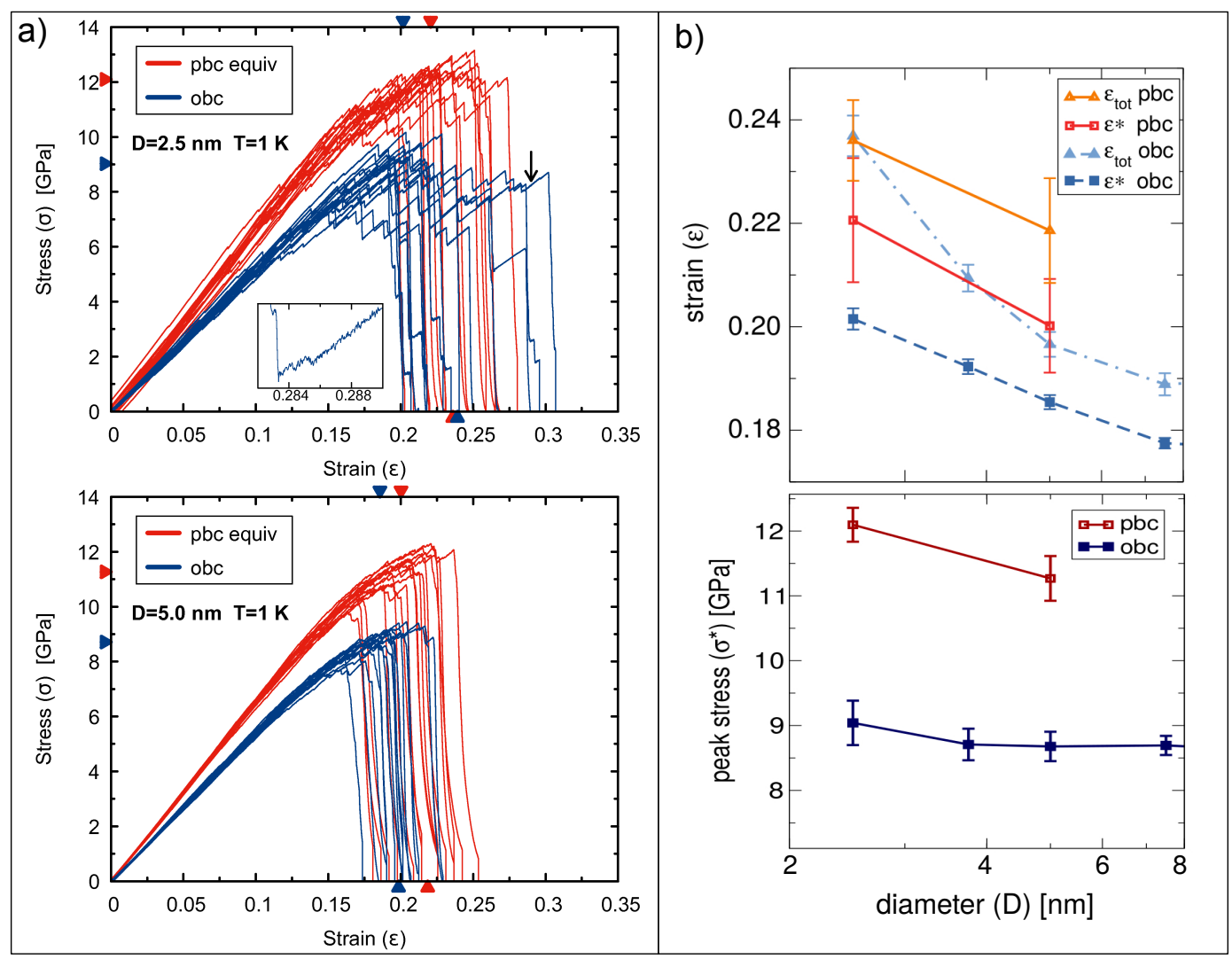

Figure 2: Obc and pbc comparison for small sample sizes at $1 \mathbf{K}-$ (a) Stress strain curves of obc and pbc samples with $D=2.5 \mathrm{~nm}$ (top panel) and $D=5 \mathrm{~nm}$ (bottom panel) at $T=1 \mathrm{~K}$. Triangles at left, top, and bottom axes report $\sigma^{*}, \epsilon^{*}$, and $\epsilon_{\text {tot }}$, respectively, averaged over 100 runs (16 shown). The inset in top panel is a magnification of the region pointed by the arrow. (b) Average values comparison as a function of size: (top panel) strain at peak $\epsilon^{*}$ and rupture strain $\epsilon_{\text {tot }}$, and (bottom panel) peak stress $\sigma^{*}$.

find indeed that even at very low temperature, atoms in the outer rim display a much larger mobility than the others. In particular, we estimate a diffusion coefficient at the surface that is from 4 to 5 times larger than in the bulk. This result is in agreement with the role of surface-induced plasticity advocated in Wingert et al. ${ }^{10}$. To corroborate this point, we analyze the behavior of the equivalent small system size samples but with periodic boundary conditions (pbc). We perform simulations of the tensile deformation of samples of length $L$ and cross-section $M \times M \equiv \pi D^{2} / 4$, with applied pbc along $x$ and $y$ directions (see Methods).

Figure 2a shows a comparison of the stress-strain curves for the $D=2.5 \mathrm{~nm}$ and $D=$ $5.0 \mathrm{~nm}$ samples at $T=1 \mathrm{~K}$ for pbc and obc. Size dependent average of $\epsilon_{\mathrm{tot}}, \epsilon^{*}$, and $\sigma^{*}$ are 


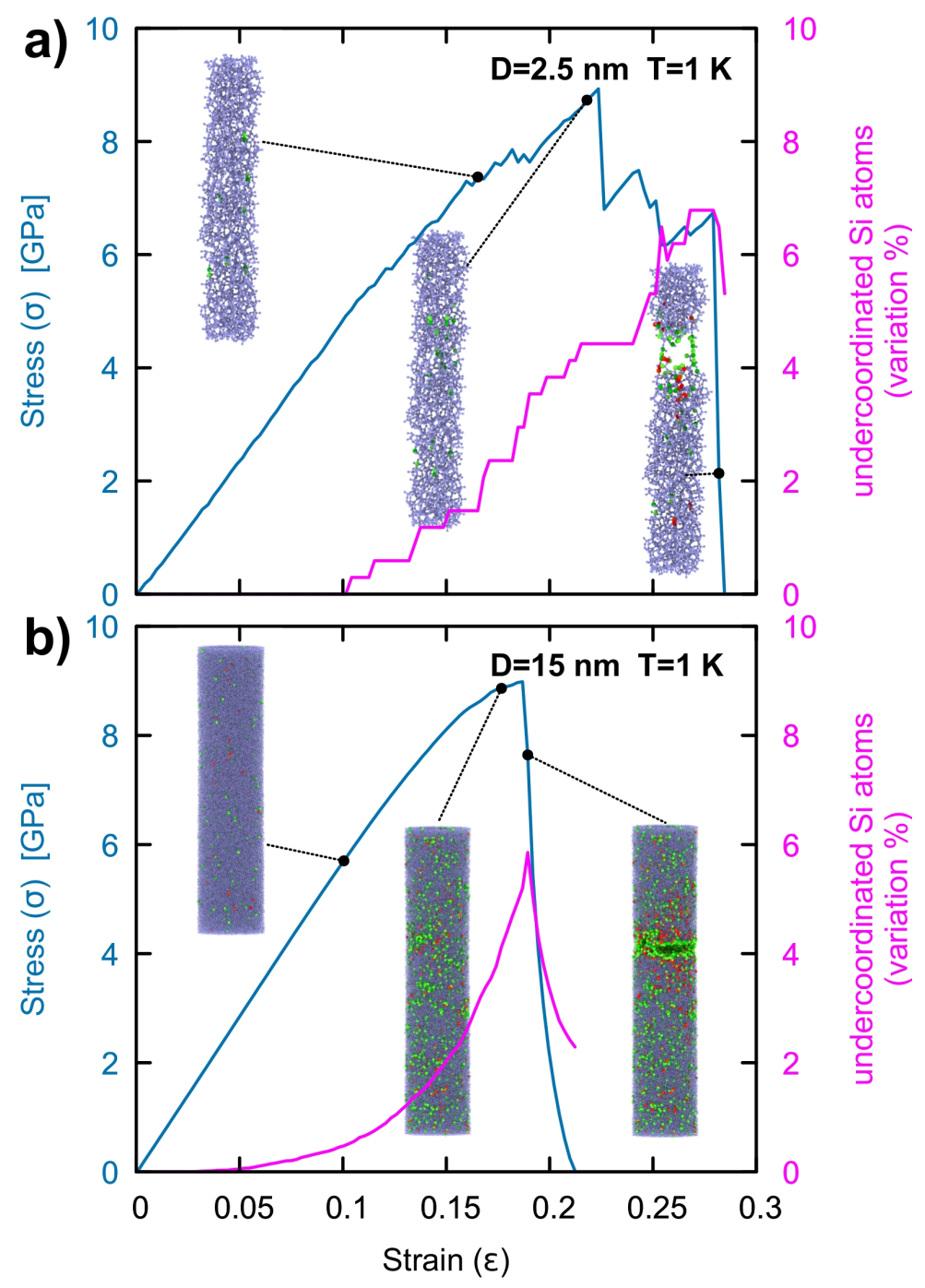

Figure 3: Coordination and damage accumulation in obc samples at $\mathbf{1} \mathbf{K}$ - Stress (blue) and percent variation of the number of undercoordinated Si atoms (magenta) as a function of the applied strain for cylindric nanofibers with (a) $D=2.5 \mathrm{~nm}$ and (b) $D=15 \mathrm{~nm}$. Snapshots report in green and red the dynamically broken and newly formed (or reformed) bonds, respectively (see SI Movie 3 and 4 for full animations).

reported in Fig. 2b. Our findings show that pbc samples present larger stress peaks, while preserving similar or even larger attainable strains $\epsilon_{\text {tot }}$. Furthermore, the smallest sample clearly displays a large plastic-like deformation regime, even when no surface is present (pbc), suggesting that ductility can not be due only to surface effects.

To investigate the origin of the observed ductile-like behavior, we inspect the evolution of the atomic coordination during deformation, a method previously used in numerical sim- 
ulations of amorphous silica, both in quiescent relaxation ${ }^{21,22}$ and tensile deformation, ${ }^{15,16}$ to reveal bond switching, an important source of stress release. ${ }^{13,14}$ A careful inspection shows that both small (Fig. 3a) and large (Fig. 3b) obc systems display similar trends in the variation of the atomic coordination. The same is observed for pbc systems. After an initial stationary period with no changes in the coordination number, roughly coincident with the purely-elastic phase of the deformation, at large-enough strain we observe a progressive decrease of well-coordinated $\mathrm{Si}^{4+}$ and $\mathrm{O}^{2-}$ atoms, compensated by an increase of under-coordinated silicon $\left(\mathrm{Si}^{3+}, \mathrm{Si}^{2+}\right.$; magenta curve in the figure) and oxygen $\left(\mathrm{O}^{1-}\right)$ atoms (see also S.I. Fig. S5). A similar mechanism is evinced from the change of the Si-O-Si angle distribution during the tensile deformation, showing a main peak at $\sim 141.5^{\circ}$ which reduces linearly at increasing applied strain, in favor of accumulation at larger angles, especially around $\sim 161^{\circ}$ (see S.I. Fig. S6).

These results provide clear evidence that damage accumulation is taking place. While these localized events might go unnoticed in the stress-strain curve of a large system, in small systems they correspond to relatively large stress drops (correlated with energy jumps, see S.I. Fig. S5). We therefore conclude that the released stress due to bond breaking is at the origin of the ductility shown by our small sized samples.

Note that some damage is already present in the unstretched samples in terms of intrinsic defects. In this respect, more defective samples are consistently shown to break earlier, being less capable of accumulating damage (see S.I. Fig. S7). A more refined structure-property relationship could be provided by the identification of polyhedral clusters in the $\mathrm{SiO}_{2}$ glass, some of which have been identified as energetically favored and thus more stable. ${ }^{23}$ The failure statistics could therefore be described, in a coarse-grained like fashion, in terms of the interaction among these hard clusters. 

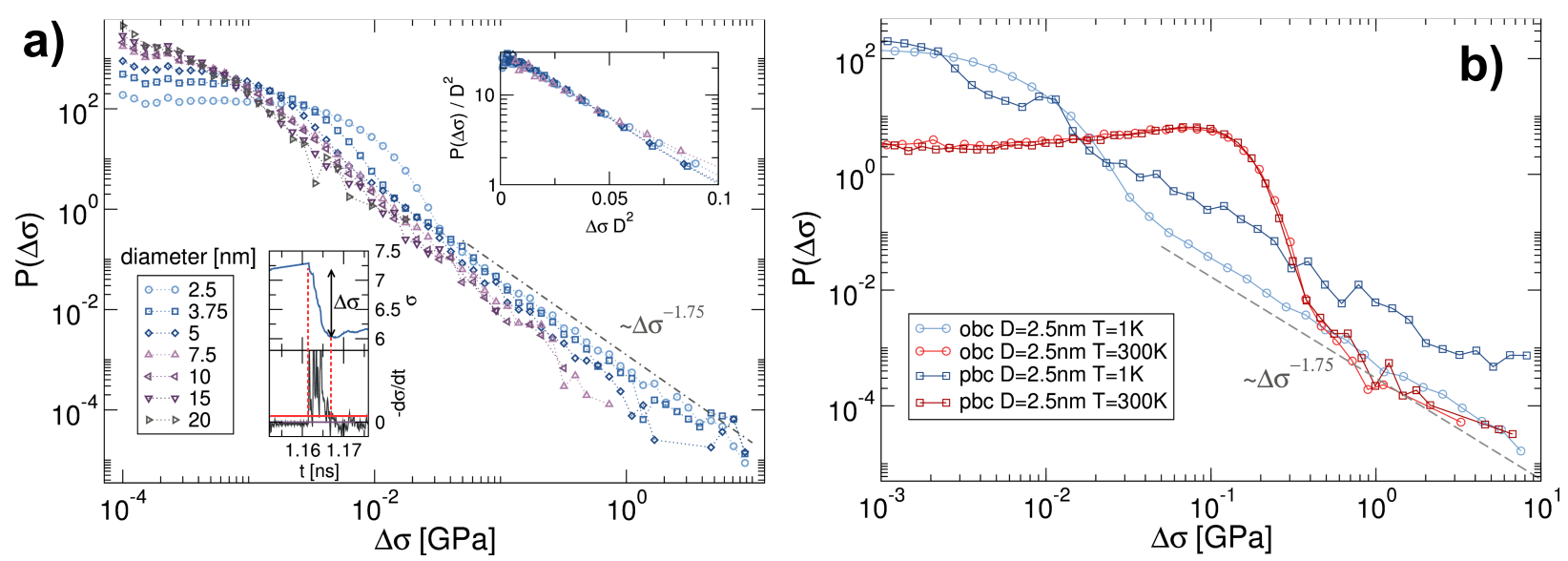

Figure 4: Stress-drops distributions - (a) $P(\Delta \sigma)$ for different sample sizes with obc. The main plot shows a power law distribution $P(\Delta \sigma) \sim \Delta \sigma^{-\tau}$ for the larger $\Delta \sigma$ with exponent $\tau \sim 1.75$; (upper inset) Lin-log plot of the $\Delta \sigma \rightarrow \Delta \sigma D^{2}$ scaling, showing a collapse of the distributions for small stress drops; (lower inset) example of stress drop and its derivative, alternative thresholds are also showed. (b) Stress-drops distribution for different temperatures and boundary conditions for the smallest system size.

\section{Stress drop statistics displays power-laws}

In order to further characterize the observed ductility, we study the statistical features of stress drops during loading. For each set of samples of a given size, we analyze the stress-time series (such as those reported in Fig.2a). After the initial elastic regime, we locate stress

drops $\Delta \sigma$ following a standard procedure: ${ }^{24-26}$ we identify time intervals where the negative time-derivative of the stress $-d \sigma / d t$ exceeds a threshold and compute the corresponding stress drops (see the lower inset of Fig. 4a for a visual explanation).

Figure 4 shows stress drop distributions for different system sizes, temperatures and boundary conditions. In the obc case, we observe a size-dependent crossover from an exponential distribution at 'small' $\Delta \sigma$ to a power-law distribution at 'large' $\Delta \sigma$ (Fig. 4a). The exponentially distributed small drops (e.g. $\Delta \sigma<3 \times 10^{-2} \mathrm{GPa}$ for the $D=2.5 \mathrm{~nm}$ case) are associated to a surface effect, since they are not present in pbc simulations (see Fig. 4b), and correspond to small and short localized rearrangements occurring during the loading phase (see the inset of Fig. 2). The upper inset of Fig. 4a reports a rescaled log-lin plot showing both the 'thermal-like' exponential distribution of these small drops and their disappearance 
as the system-size increases (the crossover to a power-law distribution moves towards smaller values of $\Delta \sigma$ as $D$ increases).

The observation of a power-law tail in $P(\Delta \sigma)$ for all system sizes suggests the presence of correlated events and collective behavior causing larger stress drops. Such an avalanche statistics associated with fracture can be understood via simple arguments based on percolation and mean-field theory. ${ }^{27}$ The deformation proceeds through localized bond failures that provoke a stress redistribution in the rest of the samples that can trigger other failure events through a cascade mechanism. An explicit correlation between stress drops and change in atomic coordination (bond breaking) is reported in S.I. Fig. S5. Notice that the power-law distribution $P(\Delta \sigma) \sim \Delta \sigma^{-\tau}$ shows up both for obc and pbc samples at low temperature (see Fig. 4b). For the system sizes and strain rate used here, it decays with the exponent is found in the range $\tau \sim 1.7-2$ depending on the conditions. We have checked that the exponent does not depend significantly on the chosen threshold (see S.I. Fig. S8). This value is somewhat larger than the mean-field exponent $\tau=3 / 2$ expected according to Shekhawat et al. ${ }^{27}$. In our interpretation the disagreement is mainly due to the very large deformation rate adopted in our simulations $\left(\dot{\epsilon}=2.5 \times 10^{8} \mathrm{~s}^{-1}\right)$, that is far away from the quasistatic conditions employed in Shekhawat et al. ${ }^{27}$. Furthermore, as shown by several works devoted to the plastic deformation of amorphous solids, ${ }^{28}$ the avalanche-related critical exponents measured in spatial models in $d=2$ and $d=3$ as well as in experiments, often differ from mean-field theory. We also measure the distribution of waiting times $T_{w}$ between stress drops, which decays as a power law $P\left(T_{w}\right) \sim T_{w}^{-\alpha}$, with an exponent $\alpha \simeq 1.5$ (see S.I. Fig. S9).

In Fig. $4 \mathrm{~b}$, we compare the cases of $T \simeq 1 \mathrm{~K}$ and $T \simeq 300 \mathrm{~K}$ for both obc and pbc. At high temperature, $P(\Delta \sigma)$ is exponential in practically all its extension and indistinguishable for pbc and obc cases, only recovering a power-law shape at its latest stage ${ }^{1}$, suggesting that the jerkiness of the stress-time series at high temperature (Fig.5) is mainly thermally induced. This observation confirms our interpretation as 'thermal-like' of the small stress discharges

\footnotetext{
${ }^{1}$ This thermal feature seems to be quite general, as has been recently observed on waiting time distributions in the bond breaking of a model gel. ${ }^{29}$
} 

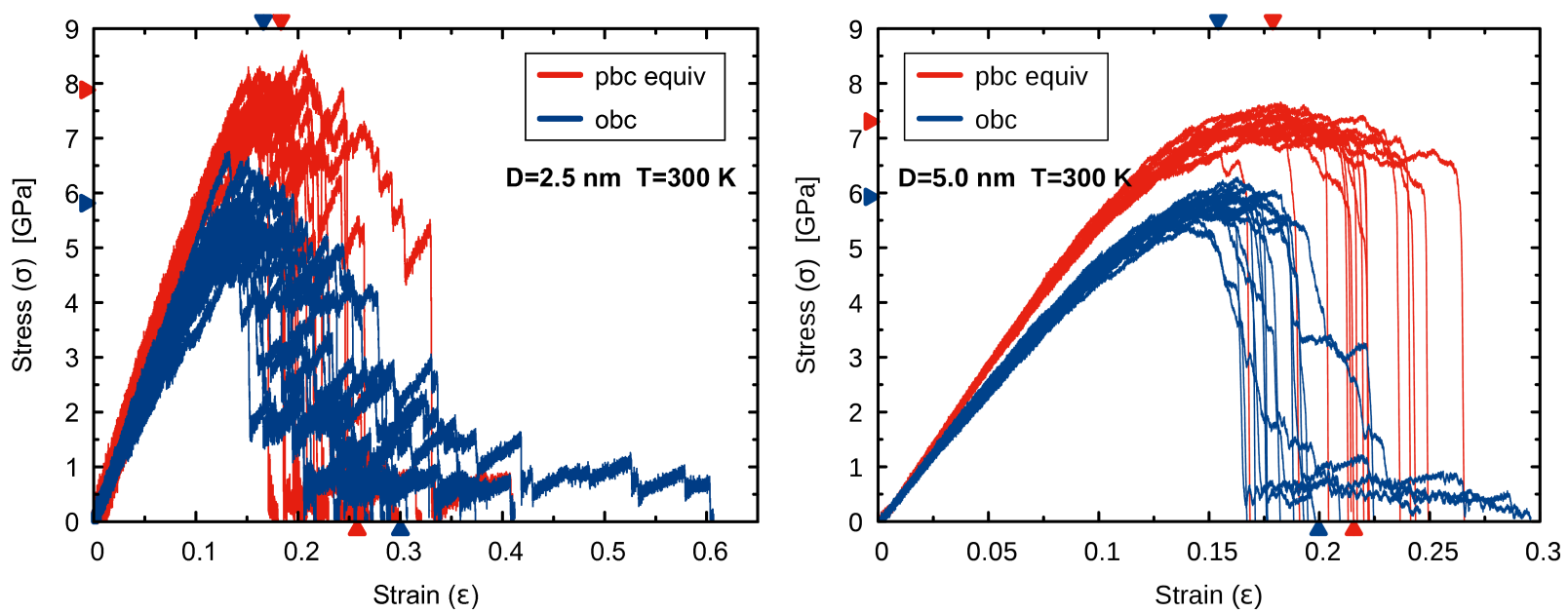

Figure 5: Stress-strain curves comparison at $300 \mathbf{K}$ - Stress strain curves of obc and pbc samples with $D=2.5 \mathrm{~nm}$ (left panel) and $D=5 \mathrm{~nm}$ (right panel) at $T=300 \mathrm{~K}$. Triangles at left, top, and bottom axes report $\sigma^{*}, \epsilon^{*}$, and $\epsilon_{\text {tot }}$, respectively, averaged over 32 runs (16 shown).

happening at $T \simeq 1 \mathrm{~K}$ for the obc pillars.

\section{Thermal effects increase ductility through necking}

Finally, to clarify the role of thermally activated events, we have performed comparative obc-pbc simulations at $T=300 \mathrm{~K}$ (see Fig. 5). When the temperature is raised in both obc and pbc, the maximum stress $\sigma^{*}$ is reduced with respect to the low temperature case (Fig. 2), owing to the reduced rigidity of the sample. On the other hand, the maximum strain $\epsilon_{\text {tot }}$ increases with temperature only for obc, while no significant changes are observed for pbc, in agreement with Pedone et al. ${ }^{30}$. The increase in strain in obc is due to re-entrant strengthening - the linear $\sigma(\epsilon)$ segments with small positive slope - signaling the occurrence of necking. Since the Young modulus is size dependent (Fig. S1), we can connect the slope of these segments to the neck thickness, showing a progressive reduction of the narrowing size until, at very low stress values, these segments become almost horizontal, meaning that a single bond is bridging the two detaching pieces of the fiber. We notice that during this process, thermal effects become more pronounced as necking starts. As a consequence, plasticity effects grow and become dominant in the last part of the process. We further note that surface effects become detrimental already at $D=5 \mathrm{~nm}$ by reducing the average $\epsilon_{\text {tot }}$ 
with respect to pbc. We expect, however, that the threshold diameter for such effect would shift toward larger $D$ at the slower strain rates typically employed in experiments.

\section{Discussion}

Amorphous silica glass represents the quintessential brittle material, failing abruptly in the elastic regime. Yet, recent experimental measurements revealed that nanoscale silica fibers become increasingly ductile as the size is decreased. ${ }^{7-12}$ The origin of this puzzling sizeinduced brittle to ductile transition is still debated with possible explanations based on surface fluidization. ${ }^{9,10}$ Indeed, experiments and simulations report evidence of a boundary layer where atoms are more mobile. ${ }^{9,10}$ Since the ratio of surface to volume increases as the sample size is decreased, it is conceivable to assume that plastic relaxation at the surface becomes more prevalent thus explaining the observed transition.

Here, we tackled the question by molecular dynamics simulations. To disentangle the individual roles of sample size and geometry from thermal induced mobility, we compared simulations with obc and pbc, performed at low and room temperature. Our main finding is that small silica glass samples are ductile even at low temperatures and with pbc, suggesting that surface fluidization can not be the only cause for the experimentally observed behavior. We observed instead that departure from brittleness in small samples is due to the diffuse bond breaking before failure. This form of damage accumulation is found with both pbc and obc, at low and room temperature.

A damage dominated size-dependent transition similar to the one observed here was analyzed theoretically in an idealized network model for the fracture of quasi-brittle disordered solids. ${ }^{27}$ According to the theory, a disordered network should always fail catastrophically in the infinite sample size limit, unless the disorder is infinitely strong in which case bonds would fail one by one as in percolation. In the more relevant case of finite disorder in a finite sample, the theory predicts a finite-size critical regime characterized by a power law 
distribution of stress drops and size dependent scaling. In small samples, failure is dominated by the coalescence of many disorder-induced cracks, but as the sample size is increased the probability to nucleate an unstable critical crack becomes larger. Hence, in large samples the effect of disorder becomes less and less relevant, leading to brittle catastrophic failure.

The theoretical scenario discussed above is in agreement with the results of our simulations, showing power law distributed avalanches, size-dependent damage accumulation and increased brittleness for larger sample sizes. The prediction of the theory do not specifically depend on the presence of a surface, in agreement with our results. Furthermore, the relevance of damage nucleation and accumulation in the failure of silica glass would be supported by in situ atomic force microscopy experiments in macroscopic samples, some of which describe the fracture propagation through the formation of nanoscale cavities ahead of the main crack. ${ }^{31,32}$ This picture was, however, questioned by other observations suggesting instead conventional brittle fracture. ${ }^{33-35}$ Regardless of the exact mechanism of fracture propagation in macroscopic samples, our results show that coalescence of accumulated damage is the triggering mechanism of fracture in nanoscale samples.

Thermal effects provide an additional layer of complexity to the landscape, increasing surface mobility and inducing sample necking, in agreement with previous work. ${ }^{9,10}$ Yet, the driver of the transition lies in the competition between disorder induced diffuse damage and stress enhancement at the tip of the cracks. Statistically larger samples will have longer cracks and therefore higher stresses at their tips, resulting in unstable crack propagation typical of brittle fracture. 


\section{Methods}

Table 1: Cylindrical samples - Size of the simulated cylindric silica glass nanofibers in terms of lenght $L$, diameter $D$, and total number of atoms $N_{a t}$. For each sample, the number $N_{\text {runs }}$ of statistical realizations is also reported.

\begin{tabular}{cccrc}
\hline \hline Sample & $L(\mathrm{~nm})$ & $D(\mathrm{~nm})$ & \multicolumn{1}{c}{$N_{a t}$} & $N_{\text {runs }}$ \\
\hline$[\mathrm{a}]$ & 10 & 2.5 & 3282 & 100 \\
{$[\mathrm{~b}]$} & 15 & 3.7 & 11076 & 100 \\
{$[\mathrm{c}]$} & 20 & 5.0 & 26250 & 100 \\
{$[\mathrm{~d}]$} & 30 & 7.5 & 87900 & 100 \\
{$[\mathrm{e}]$} & 40 & 10 & 210000 & 100 \\
{$[\mathrm{f}]$} & 60 & 15 & 708000 & 74 \\
{$[\mathrm{~g}]$} & 80 & 20 & 1680000 & 15 \\
\hline \hline
\end{tabular}

Obc samples preparation - We have considered a set of seven cylindric samples, with length $L$ ranging from 10 to $80 \mathrm{~nm}$ and diameter $D$ from 2.5 to $20 \mathrm{~nm}$, and with fixed aspect ratio $L / D=4$ (see Table 1 ). For each size, $N_{\text {runs }}$ statistically-equivalent samples have been prepared starting from $\mathrm{Si}$ and $\mathrm{O}$ atoms randomly arranged in a cylindrical box. The number of atoms was chosen to match the target density and the charge neutrality was maintained. Constraining the atoms to stay in a cylindrical configuration, a NVT quenching scheme from $4000 \mathrm{~K}$ down to $1 \mathrm{~K}$ was performed to anneal the silica liquid into glass state, with a nominal cooling rate of $50 \mathrm{~K} / \mathrm{ps}$. A time step of $1 \mathrm{fs}$ was used. Afterwards, an equilibration run was performed at $T=1 \mathrm{~K}$ for $20 \mathrm{ps}$ upon the removal of the cylindrical constraint.

Pbc samples preparation - Periodic samples have been obtained starting from an orthogonal box of size $M \times M \times L$, with square cross sectional area matching that of the corresponding cylindrical sample, and periodic boundaries along all directions. After the same annealing procedure of the obc case, we have removed the pbc along $z$ and performed an additional NPT relaxation step with cell relaxation along $x-y$ in order to minimize the lateral pressure. The same lateral barostat was applied during the tensile deformation, using the same protocol described above.

Strain - The uniaxial tensile deformation was performed at a constant strain rate of 
$\lambda=2.5 \times 10^{8} \mathrm{~s}^{-1}$, by moving the external $5 \AA$ of the sample (end caps) with constant velocity $v=\lambda L / 2$ along the axial $z$ direction, right after an initial linear velocity ramp (10 ps) with $v=a t$ where $t$ is the simulation time and $a=10^{-4} \AA / \mathrm{fs}^{2}$. The end caps atoms are free to move along the perpendicular $x-y$ directions. Temperature and pressure were controlled by Nosé-Hoover thermostat and barostat as implemented in LAMMPS, ${ }^{36}$ with applied damping time factors of $1 \mathrm{ps}$ and $10 \mathrm{ps}$, respectively.

Force fields - Atomic interactions were described by the Watanabe potential, ${ }^{37,38}$ an extension of the Stillinger-Weber (SW) potential, ${ }^{39}$ which has been proven to well reproduce the experimental features of Si-O mixed systems also under applied strain. ${ }^{37,38,40,41}$ The forcefield consists of two terms: a two-body interaction that depends on distance and a three-body interaction that describe rotational and translational symmetry. The short cutoff distances and the replacement of the usual Coulomb interaction term by a coordination-based bond softening function for Si-O atoms accounting for the environmental dependence, allow to perform computationally efficient simulations of large scale systems.

The density obtained with the Watanabe potential in a bulk system was $\rho_{0}=2.196 \mathrm{~g} / \mathrm{cm}^{3}$, well matching the experimental value of $2.2 \mathrm{~g} / \mathrm{cm}^{3} .{ }^{42}$ The effective radius $R$ of the cylindric samples, which defines the volume $V$ and thus the calculated stress $\sigma$, was chosen at $50 \%$ of the normalized radial density profile. The radial density profile of all the cylindric samples shows a smooth drop of the density at the fiber surface, with a skin size of about $0.5 \mathrm{~nm}$ (see S.I. Fig. S4). The surface roughness has been measured by considering the $N_{\text {surf }}$ most external atoms, with $N_{\text {surf }}=2 \pi R L \rho, \rho$ being the surface density fixed to 10 atoms $/ \mathrm{nm}^{2}$. Given $R_{\text {avg }}$ the average radial position - from the cylinder axis - of these $N_{\text {surf }}$ atoms, the surface roughness is calculated as standard deviation of the radial coordinate from such average: $\sigma_{s}^{2}=\left\langle\left(r-R_{\text {avg }}\right)^{2}\right\rangle$. The value $\sigma_{s}$ is averaged over 20 random-generated samples. For the $D=2.5 \mathrm{~nm}$ and $D=5 \mathrm{~nm}$ sizes, the average $\left\langle\sigma_{s}\right\rangle$ results $0.93 \pm 0.15 \AA$ and $0.90 \pm 0.05 \AA$, respectively, showing no substantial variation of the surface roughness with the nanofiber size. 


\section{Author contribution}

S.Z. designed and coordinated research; S.B., A.L.S., and R.G. performed the simulations; S.B., E.E.F., and R.G., performed data analysis and produced figures and movies; S.B., E.E.F., R.G., and S.Z. wrote the paper.

\section{Acknowledgments}

This research has been supported by ERC Advanced grant no. 291002 SIZEFFECTS.

\section{References}

(1) Alava, M. J.; Nukala, P. K. V. V.; Zapperi, S. Statistical models of fracture. Advances in Physics 2006, 55, 349-476.

(2) Lu, J.; Ravichandran, G.; Johnson, W. L. Deformation behavior of the Zr 41.2 Ti $13.8 \mathrm{Cu} 12.5 \mathrm{Ni} 10 \mathrm{Be} 22.5$ bulk metallic glass over a wide range of strain-rates and temperatures. Acta materialia 2003, 51, 3429-3443.

(3) Yue, Y.; Zheng, K. Strong strain rate effect on the plasticity of amorphous silica nanowires. Applied Physics Letters 2014, 104, 231906.

(4) Schuh, C. A.; Hufnagel, T. C.; Ramamurty, U. Mechanical behavior of amorphous alloys. Acta Materialia 2007, 55, 4067-4109.

(5) Li, G.; Jiang, M.; Jiang, F.; He, L.; Sun, J. Temperature-induced ductile-to-brittle transition of bulk metallic glasses. Applied Physics Letters 2013, 102, 171901.

(6) Yuan, F.; Huang, L. Brittle to ductile transition in densified silica glass. Scientific reports 2014,4 .

(7) Jang, D.; Greer, J. R. Transition from a strong-yet-brittle to a stronger-and-ductile state by size reduction of metallic glasses. Nature materials 2010, 9, 215-219. 
(8) Chen, D. Z.; Jang, D.; Guan, K. M.; An, Q.; Goddard, W. A.; Greer, J. R. Nanometallic Glasses: Size Reduction Brings Ductility, Surface State Drives Its Extent. Nano Letters 2013, 13, 4462-4468.

(9) Luo, J.; Wang, J.; Bitzek, E.; Huang, J. Y.; Zheng, H.; Tong, L.; Yang, Q.; Li, J.; Mao, S. X. Size-Dependent Brittle-to-Ductile Transition in Silica Glass Nanofibers. Nano Letters 2016, 16, 105-113.

(10) Wingert, M. C.; Kwon, S.; Cai, S.; Chen, R. Fluid-like Surface Layer and Its Flow Characteristics in Glassy Nanotubes. Nano Letters 2016, 16, 7545-7550.

(11) Şopu, D.; Foroughi, A.; Stoica, M.; Eckert, J. Brittle-to-Ductile Transition in Metallic Glass Nanowires. Nano Letters 2016, 16, 4467-4471.

(12) Hasan, M.; Kumar, G. High-throughput drawing and testing of metallic glass nanostructures. Nanoscale 2017, 9, 3261-3268.

(13) Chen, Y.-C.; Lu, Z.; Nomura, K.-i.; Wang, W.; Kalia, R. K.; Nakano, A.; Vashishta, P. Interaction of Voids and Nanoductility in Silica Glass. Phys. Rev. Lett. 2007, 99, 155506.

(14) Pradeep, N.; Kim, D.-I.; Grobelny, J.; Hawa, T.; Henz, B.; Zachariah, M. R. Ductility at the nanoscale: Deformation and fracture of adhesive contacts using atomic force microscopy. Applied Physics Letters 2007, 91, 203114.

(15) Tang, C.; Dávila, L. P. Anomalous surface states modify the size-dependent mechanical properties and fracture of silica nanowires. Nanotechnology 2014, 25, 435702.

(16) Tang, C.; Dávila, L. P. Strain-induced structural modifications and size-effects in silica nanowires. Journal of Applied Physics 2015, 118, 094302.

(17) Kim, Y.-J.; Lee, W. W.; Choi, I.-C.; Yoo, B.-G.; Han, S. M.; Park, H.-G.; Park, W. I.; 
Jang, J.-i. Time-dependent nanoscale plasticity of $\mathrm{ZnO}$ nanorods. Acta Materialia 2013, 61, 7180-7188.

(18) Cheng, G.; Miao, C.; Qin, Q.; Li, J.; Xu, F.; Haftbaradaran, H.; Dickey, E. C.; Gao, H.; Zhu, Y. Large anelasticity and associated energy dissipation in singlecrystalline nanowires. Nature nanotechnology 2015, 10, 687-691.

(19) Fakhraai, Z.; Forrest, J. Measuring the surface dynamics of glassy polymers. Science 2008, 319, 600-604.

(20) Chai, Y.; Salez, T.; McGraw, J. D.; Benzaquen, M.; Dalnoki-Veress, K.; Raphaël, E.; Forrest, J. A. A direct quantitative measure of surface mobility in a glassy polymer. Science 2014, 343, 994-999.

(21) Coslovich, D.; Pastore, G. Dynamics and energy landscape in a tetrahedral network glass-former: direct comparison with models of fragile liquids. Journal of Physics: Condensed Matter 2009, 21, 285107.

(22) Horbach, J.; Kob, W.; Binder, K.; Angell, C. A. Finite size effects in simulations of glass dynamics. Physical Review E 1996, 54, R5897.

(23) Harkless, J. A. W.; Stillinger, D. K.; Stillinger, F. H. Structures and Energies of $\mathrm{SiO}_{2}$ Clusters. J. Phys. Chem. 1996, 100, 1098-1103.

(24) Laurson, L.; Illa, X.; Santucci, S.; Tallakstad, K. T.; Maloy, K. J.; Alava, M. J. Evolution of the average avalanche shape with the universality class. Nature Communications 2013,4 .

(25) Liu, C.; Ferrero, E. E.; Puosi, F.; Barrat, J.-L.; Martens, K. Driving Rate Dependence of Avalanche Statistics and Shapes at the Yielding Transition. Phys. Rev. Lett. 2016, $116,065501$. 
(26) Janićević, S.; Laurson, L.; Måløy, K. J.; Santucci, S.; Alava, M. J. Interevent Correlations from Avalanches Hiding Below the Detection Threshold. Phys. Rev. Lett. 2016, $117,230601$.

(27) Shekhawat, A.; Zapperi, S.; Sethna, J. P. From Damage Percolation to Crack Nucleation Through Finite Size Criticality. Phys. Rev. Lett. 2013, 110, 185505.

(28) Nicolas, A.; Ferrero, E. E.; Martens, K.; Barrat, J.-L. Deformation and flow of amorphous solids: a review of mesoscale elastoplastic models. arXiv preprint arXiv:1708.09194 2017,

(29) Bouzid, M.; Colombo, J.; Barbosa, L. V.; Del Gado, E. Elastically driven intermittent microscopic dynamics in soft solids. Nature communications 2017, 8, ncomms15846.

(30) Pedone, A.; Malavasi, G.; Menziani, M. C.; Segre, U.; Cormack, A. N. Molecular dynamics studies of stress- strain behavior of silica glass under a tensile load. Chemistry of Materials 2008, 20, 4356-4366.

(31) Célarié, F.; Prades, S.; Bonamy, D.; Ferrero, L.; Bouchaud, E.; Guillot, C.; Marlière, C. Glass Breaks like Metal, but at the Nanometer Scale. Phys. Rev. Lett. 2003, 90, 075504.

(32) Bonamy, D.; Prades, S.; Rountree, C. L.; Ponson, L.; Dalmas, D.; Bouchaud, E.; RaviChandar, K.; Guillot, C. Nanoscale damage during fracture in silica glass. International Journal of Fracture 2006, 140, 3-14.

(33) Guin, J. P.; Wiederhorn, S. M. Fracture of silicate glasses: Ductile or brittle? Phys. Rev. Lett. 2004, 92, 215502.

(34) López-Cepero, J. M.; Wiederhorn, S. M.; Fett, T.; Guin, J. P. Do plastic zones form at crack tips in silicate glasses? Int. J. Mater. Res. 2007, 98, 1170-1176. 
(35) Fett, T.; Rizzi, G.; Creek, D.; Wagner, S.; Guin, J. P.; López-Cepero, J. M.; Wiederhorn, S. M. Finite element analysis of a crack tip in silicate glass: No evidence for a plastic zone. Phys. Rev. B 2008, 77, 174110.

(36) Plimpton, S. Fast parallel algorithms for short-range molecular dynamics. Journal of computational physics 1995, 117, 1-19.

(37) Watanabe, T.; Fujiwara, H.; Noguchi, H.; Hoshino, T.; Ohdomari, I. Novel interatomic potential energy function for Si, O mixed systems. Japanese journal of applied physics 1999, 38, L366.

(38) Watanabe, T.; Yamasaki, D.; Tatsumura, K.; Ohdomari, I. Improved interatomic potential for stressed Si, O mixed systems. Applied surface science 2004, 234, 207-213.

(39) Stillinger, F. H.; Weber, T. A. Computer simulation of local order in condensed phases of silicon. Physical review B 1985, 31, 5262.

(40) Dalla Torre, J.; Bocquet, J.-L.; Limoge, Y.; Crocombette, J.-P.; Adam, E.; Martin, G.; Baron, T.; Rivallin, P.; Mur, P. Study of self-limiting oxidation of silicon nanoclusters by atomistic simulations. Journal of Applied Physics 2002, 92, 1084-1094.

(41) Djurabekova, F.; Nordlund, K. Atomistic simulation of the interface structure of Si nanocrystals embedded in amorphous silica. Physical Review B 2008, 7r, 115325.

(42) Brückner, R. Properties and structure of vitreous silica. II. Journal of non-crystalline solids 1970, 5, 177-216. 
Graphical TOC Entry

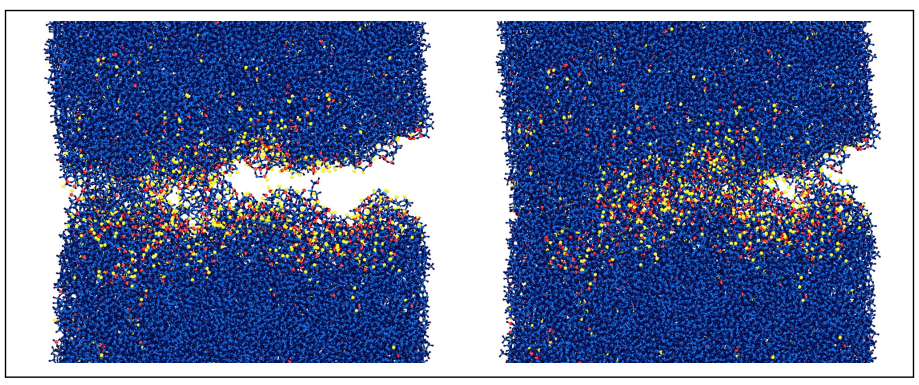

